\title{
NOISE AND ELECTROMAGNETIC FIELD MEASUREMENTS FOR THE MERCHANT SHIP ALBATROS
}

\author{
Gheorghe SAMOILESCU*, Adelina BORDIANU**, \\ Serghei RADU***, Alina BARBU**** \\ * “Mircea cel Bătrân” Naval Academy, Constanța, Romania, \\ $* *$ Polytechnic University of Bucharest, Romania,

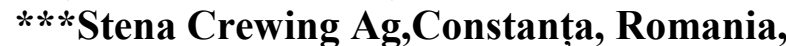 \\ **** Constanța Maritime University, Romania \\ samoilescugheorghe@yahoo.com, adelina_vb@yahoo.com, \\ sergradu@yahoo.com, alucu.71@yahoo.com
}

\begin{abstract}
The paper analyses the results of performed measurements regarding the density of electromagnetic power at various points on board a ship, the noise level determined by the operation of certain broadcasting stations and the intensity of the radiated electric field by a station of $250 \mathrm{MHz}$. We have presented thereby the sources triggering natural and artificial electromagnetic disturbances with their pertaining components.
\end{abstract}

Keywords: power density, intensity of electric field, broadcasting station, disturbing sources, noise levels

\section{Introduction}

The equipment of a ship with telecommunication, navigation, armament, radio transmitter, radar and power subsystems requires a full evaluation of electromagnetic compatibility problems incurred thereby. Electromagnetic compatibility is a feature specific to an electric unit enabling the latter to operate at normal parameters in its own electromagnetic environment without influencing this specific environment. [1,8]

The units refer to equipments and subsystems. Provided these units belong to the same system, we then have an internal electromagnetic compatibility.

Due to the diverse range of electric and electronic devices used onboard ships, as well as to their power enhancement, there has been an alarming increase in abnormalities, consisting of component wreckage, breakdowns or malfunctions during operation of circuits or equipments, affecting the personnel phenomena could not be ignored any longer without deliberately taking some risks, with negative effects on the ship, and on the seagoing personnel as well.

Therefore, we have started some research on the disturbing electromagnetic interferences aiming at highlighting the generating sources, their transmission paths, and the effects they have on electronic apparatus and the navigating personnel, with a view to finally enable us to devise adequate antidisturbing technologies. [2, 5, 6, 7, 8, 10]

A first step would be identifying and setting up specifications for each type 
of electromagnetic disturbances onboard. These disturbances may be classified according to their nature, the transmission paths, the place of occurrence, and the distance between the source and the perturbed.

According to their nature, electromagnetic disturbances may be artificial and natural. [3, 4, 9] In their turn, artificial disturbances may be divided into unintentional and intentional disturbances. From the category of unintentional disturbances we may name the following $[1,2]$ :

- the electromagnetic disturbances produced by short-circuits and transitory statuses of the feeding and operating gear;

- the electromagnetic disturbances produced by power electronics due to the phenomenon of commutation;

- the electromagnetic disturbances produced by electrostatic discharges;

- the electromagnetic disturbances produced by operation of radio units, radar, and navigation devices.

Among the intentional disturbances, the following are worth mentioning: the electronic interference and the electromagnetic impulse of the impulse weapon.

At present, the wide range of topics approached in the update field of electromagnetic compatibility is impressive, and it comprises [4, 9]:

a. sources of electromagnetic interference (electric and electronic units, electrostatic discharges);

b. specific values for the electric units involved by electromagnetic interferences and their respective parameters: levels and variation speeds for $\overline{\mathrm{H}}, \overline{\mathrm{E}}, \overline{\mathrm{S}}$ (the power density of the field), U, I, frequency, type of modulation, polarization, wave shape, distortion factor etc;

c. paths of transmission or propagation of parasite signals among equipment, systems, cables and the outer environment;

d. methods and techniques of measurements and testing the electromagnetic interference phenomena;

e. control of antenna electromagnetic interferences (stationary, rotating, with straight bundle) of $\mathrm{HF}$, VHF, UHF;

f. techniques of anti-disturbing protection; screening, filtering, linkage to the electronic mass and the ground (the ship's hull);

g. protection of the human factor on board ships (biological effects of electromagnetic interferences).

\section{Measurement of the electromagnetic power density radiated by the quarter deck of the 8000 dwt Cargo ship Albatros [11]}

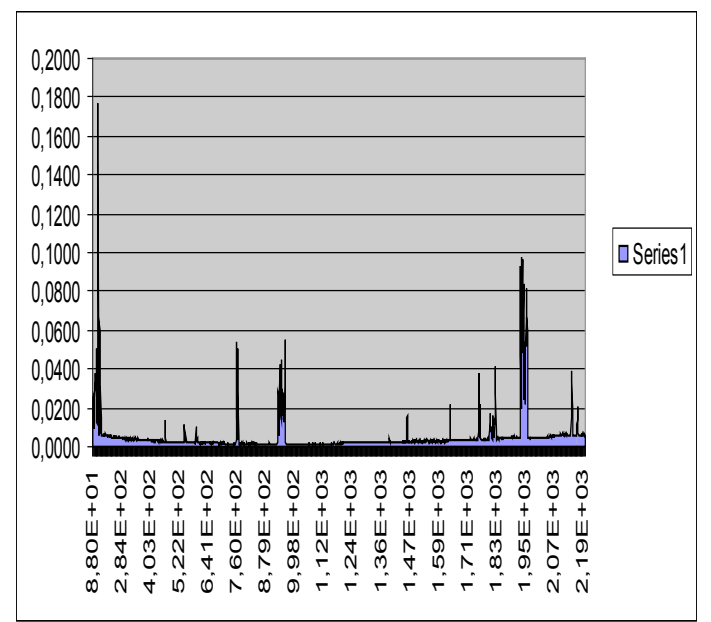

Fig.1 Measurement of the environmental noise level at 09:52 with emission of the JR civilian unit on board Albatros on channel 4 VHF (frequency de 156,200 $\mathrm{MHz}$ ) modulated with a $25 \mathrm{~W}$ emission power - table 1.

Table 1. Main measured values, fig.1.

\begin{tabular}{|c|c|c|}
\hline $\begin{array}{c}\text { Frequency } \\
{[\mathrm{MHz}]}\end{array}$ & $\begin{array}{c}\text { Field } \\
\text { Strength } \\
{[\mathrm{V} / \mathrm{m}]}\end{array}$ & $\begin{array}{c}\text { Power } \\
\text { Density } \\
{[\mu \mathrm{W} / \mathrm{cm} 2]}\end{array}$ \\
\hline $1.56 \mathrm{E}+02$ & 0.1766 & 0.0083 \\
\hline $1.57 \mathrm{E}+02$ & 0.1511 & 0.0061 \\
\hline $1.94 \mathrm{E}+03$ & 0.0979 & 0.0025 \\
\hline
\end{tabular}




\begin{tabular}{|c|c|c|}
\hline $1.94 \mathrm{E}+03$ & 0.0963 & 0.0025 \\
\hline $1.55 \mathrm{E}+02$ & 0.0948 & 0.0024 \\
\hline $1.93 \mathrm{E}+03$ & 0.0927 & 0.0023 \\
\hline $1.94 \mathrm{E}+03$ & 0.0844 & 0.0019 \\
\hline $1.96 \mathrm{E}+03$ & 0.0821 & 0.0018 \\
\hline $1.94 \mathrm{E}+03$ & 0.0789 & 0.0017 \\
\hline $1.94 \mathrm{E}+03$ & 0.0757 & 0.0015 \\
\hline $1.93 \mathrm{E}+03$ & 0.0717 & 0.0014 \\
\hline $1.96 \mathrm{E}+03$ & 0.0669 & 0.0012 \\
\hline $1.96 \mathrm{E}+03$ & 0.0655 & 0.0011 \\
\hline $1.95 \mathrm{E}+03$ & 0.0649 & 0.0011 \\
\hline $1.91 \mathrm{E}+02$ & 0.0643 & 0.0011 \\
\hline $1.95 \mathrm{E}+03$ & 0.0627 & 0.0010 \\
\hline
\end{tabular}

\begin{tabular}{|c|c|c|}
\hline $1.04 \mathrm{E}+02$ & 0.0735 & 0.0014 \\
\hline $1.95 \mathrm{E}+03$ & 0.0735 & 0.0014 \\
\hline $1.96 \mathrm{E}+03$ & 0.0714 & 0.0014 \\
\hline $1.55 \mathrm{E}+02$ & 0.0706 & 0.0013 \\
\hline
\end{tabular}

3. Measurement of the electromagnetic power density radiated by the units involved in the measurement procedures

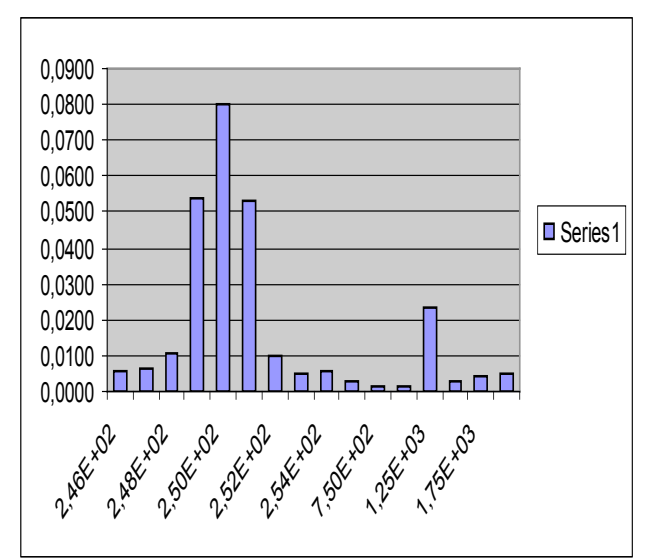

Fig.3. Measurement of radiated electromagnetic power density by a unit on a frequency of $250 \mathrm{MHz}$, modulated, for the nominal power of emission

Fig. 2. Measurement of the environmental noise level at 10:10 with emission of the JR civilian unit on board Albatros on channel 4 VHF (frequency de 156.200 $\mathrm{MHz}$ ) modulated with a $25 \mathrm{~W}$ emission power

Table 2. Main measured values, fig. 2.

\begin{tabular}{|c|c|c|}
\hline $\begin{array}{c}\text { Frequency } \\
{[\mathrm{MHz}]}\end{array}$ & $\begin{array}{c}\text { Field } \\
\text { Strength } \\
{[\mathrm{V} / \mathrm{m}]}\end{array}$ & $\begin{array}{c}\text { Power } \\
\text { Density } \\
{[\mu \mathrm{W} / \mathrm{cm} 2]}\end{array}$ \\
\hline $1.56 \mathrm{E}+02$ & 0.1324 & 0.0046 \\
\hline $1.57 \mathrm{E}+02$ & 0.1128 & 0.0034 \\
\hline $1.95 \mathrm{E}+03$ & 0.1092 & 0.0032 \\
\hline $1.95 \mathrm{E}+03$ & 0.1036 & 0.0028 \\
\hline $1.93 \mathrm{E}+03$ & 0.0961 & 0.0025 \\
\hline $1.94 \mathrm{E}+03$ & 0.0931 & 0.0023 \\
\hline $1.94 \mathrm{E}+03$ & 0.0897 & 0.0021 \\
\hline $1.94 \mathrm{E}+03$ & 0.0858 & 0.0020 \\
\hline $1.95 \mathrm{E}+03$ & 0.0795 & 0.0017 \\
\hline $1.95 \mathrm{E}+03$ & 0.0774 & 0.0016 \\
\hline $1.94 \mathrm{E}+03$ & 0.0772 & 0.0016 \\
\hline $1.93 \mathrm{E}+03$ & 0.0761 & 0.0015 \\
\hline
\end{tabular}

Table3. Main measured values fig. 3 .

\begin{tabular}{|c|c|c|c|}
\hline $\begin{array}{c}\text { Nr. of } \\
\text { harmonics }\end{array}$ & $\begin{array}{c}\text { Frequency } \\
{[\mathrm{MHz}]}\end{array}$ & $\begin{array}{c}\text { Field } \\
\text { Strength } \\
{[\mathrm{V} / \mathrm{m}]}\end{array}$ & $\begin{array}{c}\text { Power } \\
\text { Density } \\
{[\mu \mathrm{W} / \mathrm{cm} 2]}\end{array}$ \\
\hline- & $2.46 \mathrm{E}+02$ & 0.0055 & 0.0000 \\
\hline- & $2.47 \mathrm{E}+02$ & 0.0061 & 0.0000 \\
\hline- & $2.48 \mathrm{E}+02$ & 0.0109 & 0.0000 \\
\hline- & $2.49 \mathrm{E}+02$ & 0.0538 & 0.0008 \\
\hline 0 & $2.50 \mathrm{E}+02$ & 0.0802 & 0.0017 \\
\hline- & $2.51 \mathrm{E}+02$ & 0.0534 & 0.0008 \\
\hline- & $2.52 \mathrm{E}+02$ & 0.0102 & 0.0000 \\
\hline- & $2.53 \mathrm{E}+02$ & 0.0052 & 0.0000 \\
\hline- & $2.54 \mathrm{E}+02$ & 0.0058 & 0.0000 \\
\hline 2 & $5.00 \mathrm{E}+02$ & 0.0025 & 0.0000 \\
\hline 3 & $7.50 \mathrm{E}+02$ & 0.0015 & 0.0000 \\
\hline 4 & $1.00 \mathrm{E}+03$ & 0.0014 & 0.0000 \\
\hline 5 & $1.25 \mathrm{E}+03$ & 0.0234 & 0.0001 \\
\hline 6 & $1.50 \mathrm{E}+03$ & 0.0029 & 0.0000 \\
\hline 7 & $1.75 \mathrm{E}+03$ & 0.0040 & 0.0000 \\
\hline 8 & $2.00 \mathrm{E}+03$ & 0.0052 & 0.0000 \\
\hline & & & \\
\hline
\end{tabular}

Table4. Main measured values, fig. 4. 


\begin{tabular}{|c|c|c|c|}
\hline $\begin{array}{c}\text { Nr. of } \\
\text { harmonics }\end{array}$ & $\begin{array}{c}\text { Frequency } \\
{[\mathrm{MHz}]}\end{array}$ & $\begin{array}{c}\text { Field } \\
\text { Strength } \\
{[\mathrm{V} / \mathrm{m}]}\end{array}$ & $\begin{array}{c}\text { Power } \\
\text { Density } \\
{[\mu \mathrm{W} / \mathrm{cm} 2]}\end{array}$ \\
\hline- & $2.47 \mathrm{E}+02$ & 0.0054 & 0.0000 \\
\hline- & $2.48 \mathrm{E}+02$ & 0.0058 & 0.0000 \\
\hline- & $2.49 \mathrm{E}+02$ & 0.0121 & 0.0000 \\
\hline 0 & $2.50 \mathrm{E}+02$ & 0.0170 & 0.0001 \\
\hline- & $2.51 \mathrm{E}+02$ & 0.0113 & 0.0000 \\
\hline- & $2.52 \mathrm{E}+02$ & 0.0050 & 0.0000 \\
\hline- & $2.53 \mathrm{E}+02$ & 0.0055 & 0.0000 \\
\hline 2 & $5.00 \mathrm{E}+02$ & 0.0019 & 0.0000 \\
\hline 3 & $7.50 \mathrm{E}+02$ & 0.0014 & 0.0000 \\
\hline 4 & $1.00 \mathrm{E}+03$ & 0.0013 & 0.0000 \\
\hline 5 & $1.25 \mathrm{E}+03$ & 0.0038 & 0.0000 \\
\hline 6 & $1.50 \mathrm{E}+03$ & 0.0030 & 0,0000 \\
\hline 7 & $1.75 \mathrm{E}+03$ & 0.0040 & 0.0000 \\
\hline 8 & $2.00 \mathrm{E}+03$ & 0.0049 & 0.0000 \\
\hline
\end{tabular}

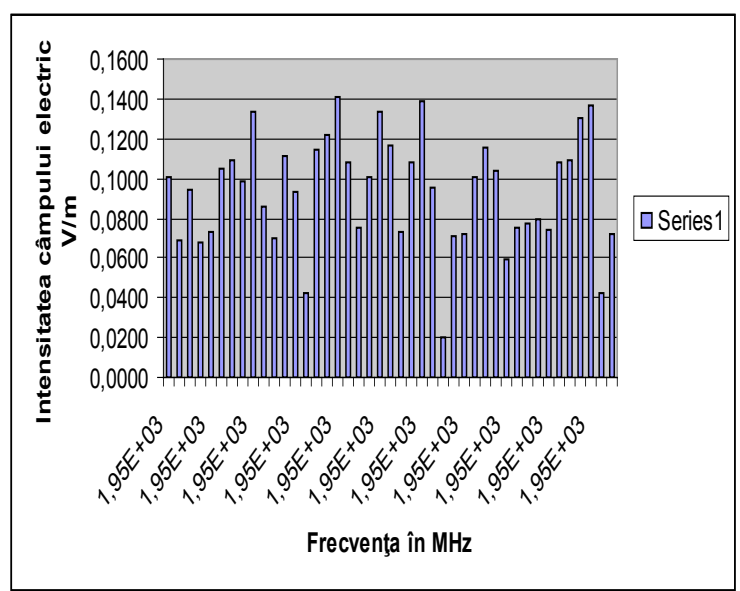

Fig. 5. Illustration of daytime measured values of a radiated electric field intensity for a frequency of $1,95 \mathrm{GHz}$

\begin{tabular}{|c|c|c|c|c|}
\hline $\begin{array}{l}\text { Freque } \\
\text { ncy in } \\
\mathrm{MHz}\end{array}$ & $\begin{array}{c}\text { Intensity } \\
\mathrm{cp} . \text { El.in } \\
\mathrm{V} / \mathrm{m}\end{array}$ & $E$ in $\mathrm{dB}$ & $\begin{array}{l}\text { Power } \\
\text { density }\end{array}$ & $\mathrm{DP}$ in $\mathrm{dB}$ \\
\hline $\begin{array}{c}1.95 \mathrm{E}+ \\
03\end{array}$ & 0.1006 & $\begin{array}{c}- \\
19.94598 \\
415\end{array}$ & 0.0027 & -25.7094 \\
\hline $\begin{array}{c}1.95 \mathrm{E}+ \\
03\end{array}$ & 0.0686 & $\begin{array}{c}- \\
23.27549 \\
608 \\
\end{array}$ & 0.0012 & -29.0389 \\
\hline $\begin{array}{c}1.95 \mathrm{E}+ \\
03\end{array}$ & 0.0948 & $\begin{array}{c}- \\
20.46644 \\
007\end{array}$ & 0.0024 & -26.2299 \\
\hline $\begin{array}{c}1.95 \mathrm{E}+ \\
03\end{array}$ & 0.0676 & $\begin{array}{c}- \\
23.39891 \\
229\end{array}$ & 0.0012 & -29.1623 \\
\hline $\begin{array}{c}1.95 \mathrm{E}+ \\
03\end{array}$ & 0.0728 & $\begin{array}{c}- \\
22.76192 \\
41\end{array}$ & 0.0014 & -28.5253 \\
\hline $\begin{array}{c}1.95 \mathrm{E}+ \\
03\end{array}$ & 0.1048 & $\begin{array}{c}- \\
19.59138 \\
534 \\
\end{array}$ & 0.0029 & -25.3548 \\
\hline $\begin{array}{c}1.95 \mathrm{E}+ \\
03\end{array}$ & 0.1092 & $\begin{array}{c}- \\
19.23724 \\
112 \\
\end{array}$ & 0.0032 & -25.0007 \\
\hline $\begin{array}{c}1.95 \mathrm{E}+ \\
03\end{array}$ & 0.0982 & $\begin{array}{c}- \\
20.15404 \\
473 \\
\end{array}$ & 0.0026 & -25.9175 \\
\hline $\begin{array}{c}1.95 \mathrm{E}+ \\
03\end{array}$ & 0.1333 & $\begin{array}{c}- \\
17.50241 \\
192 \\
\end{array}$ & 0.0047 & -23.2658 \\
\hline $\begin{array}{c}1.95 \mathrm{E}+ \\
03\end{array}$ & 0.0863 & $\begin{array}{c}- \\
21.28299 \\
064\end{array}$ & 0.0020 & -27.0464 \\
\hline $\begin{array}{c}1.95 \mathrm{E}+ \\
03\end{array}$ & 0.0704 & $\begin{array}{c}- \\
23.05359 \\
816 \\
\end{array}$ & 0.0013 & -28.817 \\
\hline $\begin{array}{c}1.95 \mathrm{E}+ \\
03\end{array}$ & 0.1114 & $\begin{array}{c}- \\
19.06107 \\
225\end{array}$ & 0.0033 & -24.8245 \\
\hline
\end{tabular}




\begin{tabular}{|c|c|c|c|c|}
\hline $\begin{array}{l}\text { Freque } \\
\text { ncy in } \\
\mathrm{MHz}\end{array}$ & $\begin{array}{c}\text { Intensity } \\
\mathrm{cp} \text {. El.in } \\
\mathrm{V} / \mathrm{m}\end{array}$ & $E$ in $\mathrm{dB}$ & $\begin{array}{l}\text { Power } \\
\text { density }\end{array}$ & $\mathrm{DP}$ in $\mathrm{dB}$ \\
\hline $\begin{array}{c}1.95 \mathrm{E}+ \\
03\end{array}$ & 0.0931 & $\begin{array}{c}- \\
20.62181 \\
934 \\
\end{array}$ & 0.0023 & -26.3852 \\
\hline $\begin{array}{c}1.95 \mathrm{E}+ \\
03\end{array}$ & 0.0423 & $\begin{array}{c}- \\
27.46717 \\
938\end{array}$ & 0.0005 & -33.2306 \\
\hline $\begin{array}{c}1.95 \mathrm{E}+ \\
03\end{array}$ & 0.1145 & $\begin{array}{c}- \\
18.82024 \\
077\end{array}$ & 0.0035 & -24.5837 \\
\hline $\begin{array}{c}1.95 \mathrm{E}+ \\
03\end{array}$ & 0.1217 & $\begin{array}{c}- \\
18.29713 \\
405\end{array}$ & 0.0039 & -24.0605 \\
\hline $\begin{array}{c}1.95 \mathrm{E}+ \\
03\end{array}$ & 0.1409 & $\begin{array}{c}- \\
17.01915 \\
664 \\
\end{array}$ & 0.0053 & -22.7826 \\
\hline $\begin{array}{c}1.95 \mathrm{E}+ \\
03\end{array}$ & 0.1082 & $\begin{array}{c}- \\
19.31274 \\
052\end{array}$ & 0.0031 & -25.0762 \\
\hline $\begin{array}{c}1.95 \mathrm{E}+ \\
03\end{array}$ & 0.0754 & $\begin{array}{l}- \\
22.45607 \\
846\end{array}$ & 0.0015 & -28.2195 \\
\hline $\begin{array}{c}1.95 \mathrm{E}+ \\
03\end{array}$ & 0.1008 & $\begin{array}{c}- \\
19.93366 \\
882 \\
\end{array}$ & 0.0027 & -25.6971 \\
\hline $\begin{array}{c}1.95 \mathrm{E}+ \\
03\end{array}$ & 0.1335 & $\begin{array}{c}- \\
17.48780 \\
438 \\
\end{array}$ & 0.0047 & -23.2512 \\
\hline $\begin{array}{c}1.95 \mathrm{E}+ \\
03\end{array}$ & 0.1169 & $\begin{array}{c}- \\
18.64144 \\
452 \\
\end{array}$ & 0.0036 & -24.4049 \\
\hline $\begin{array}{c}1.95 \mathrm{E}+ \\
03\end{array}$ & 0.0731 & $\begin{array}{c}- \\
22.72336 \\
186 \\
\end{array}$ & 0.0014 & -28.4868 \\
\hline $\begin{array}{c}1.95 \mathrm{E}+ \\
03\end{array}$ & 0.1084 & $\begin{array}{c}- \\
19.29678 \\
863 \\
\end{array}$ & 0.0031 & -25.0602 \\
\hline $\begin{array}{c}1.95 \mathrm{E}+ \\
03\end{array}$ & 0.1383 & $\begin{array}{c}- \\
17.18397 \\
169\end{array}$ & 0.0051 & -22.9474 \\
\hline $\begin{array}{c}1.95 \mathrm{E}+ \\
03\end{array}$ & 0.0954 & $\begin{array}{c}- \\
20.40711 \\
4 \\
\end{array}$ & 0.0024 & -26.1705 \\
\hline $\begin{array}{c}1.95 \mathrm{E}+ \\
03\end{array}$ & 0.0202 & $\begin{array}{c}- \\
33.88362 \\
94\end{array}$ & 0.0001 & -39.647 \\
\hline $\begin{array}{c}1.95 \mathrm{E}+ \\
03\end{array}$ & 0.0715 & $\begin{array}{c}- \\
22.91108 \\
384 \\
\end{array}$ & 0.0014 & -28.6745 \\
\hline $\begin{array}{c}1.95 \mathrm{E}+ \\
03\end{array}$ & 0.0718 & $\begin{array}{c}- \\
22.88064 \\
756\end{array}$ & 0.0014 & -28.6441 \\
\hline $\begin{array}{c}1,95 \mathrm{E}+ \\
03\end{array}$ & 0.1009 & $\begin{array}{c}- \\
19.92425 \\
631\end{array}$ & 0.0027 & -25.6877 \\
\hline $1.95 \mathrm{E}+$ & 0.1156 & - & 0.0035 & -24.5028 \\
\hline
\end{tabular}

\begin{tabular}{|c|c|c|c|c|}
\hline $\begin{array}{l}\text { Freque } \\
\text { ncy in } \\
\mathrm{MHz}\end{array}$ & $\begin{array}{l}\text { Intensity } \\
\mathrm{cp} . \text { El.in } \\
\mathrm{V} / \mathrm{m}\end{array}$ & $E$ in $\mathrm{dB}$ & $\begin{array}{l}\text { Power } \\
\text { density }\end{array}$ & $\mathrm{DP}$ in $\mathrm{dB}$ \\
\hline 03 & & $\begin{array}{c}18.73933 \\
858\end{array}$ & & \\
\hline $\begin{array}{c}1.95 \\
E+03\end{array}$ & 0.1035 & $\begin{array}{c}- \\
19.69815 \\
031\end{array}$ & 0.0028 & -25.4616 \\
\hline $\begin{array}{c}1.95 \mathrm{E}+ \\
03\end{array}$ & 0.0596 & $\begin{array}{c}- \\
24.49497 \\
106\end{array}$ & 0.0009 & -30.2584 \\
\hline $\begin{array}{c}1.95 \mathrm{E}+ \\
03\end{array}$ & 0.0758 & $\begin{array}{c}- \\
22.41149 \\
497\end{array}$ & 0.0015 & -28.1749 \\
\hline $\begin{array}{c}1.95 \mathrm{E}+ \\
03\end{array}$ & 0.0776 & $\begin{array}{c}- \\
22.20678 \\
553\end{array}$ & 0.0016 & -27.9702 \\
\hline $\begin{array}{c}1.95 \mathrm{E}+ \\
03\end{array}$ & 0.0797 & $\begin{array}{c}- \\
21.97575 \\
12 \\
\end{array}$ & 0.0017 & -27.7392 \\
\hline $\begin{array}{c}1,95 \mathrm{E}+ \\
03\end{array}$ & 0.0737 & $\begin{array}{c}- \\
22.65149 \\
646\end{array}$ & 0.0014 & -28.4149 \\
\hline $\begin{array}{c}1.95 \mathrm{E}+ \\
03\end{array}$ & 0.1079 & $\begin{array}{c}- \\
19.33912 \\
481\end{array}$ & 0.0031 & -25.1025 \\
\hline $\begin{array}{c}1.95 \mathrm{E}+ \\
03\end{array}$ & 0.1094 & $\begin{array}{c}- \\
19.22119 \\
502\end{array}$ & 0.0032 & -24.9846 \\
\hline $\begin{array}{c}1.95 \mathrm{E}+ \\
03\end{array}$ & 0.1301 & $\begin{array}{c}- \\
17.71551 \\
915 \\
\end{array}$ & 0.0045 & -23.4789 \\
\hline $\begin{array}{c}1.95 \mathrm{E}+ \\
03\end{array}$ & 0.1365 & $\begin{array}{c}- \\
17.29484 \\
095 \\
\end{array}$ & 0.0049 & -23.0583 \\
\hline $\begin{array}{c}1.95 \mathrm{E}+ \\
03\end{array}$ & 0.0425 & $\begin{array}{c}- \\
27.42391 \\
188 \\
\end{array}$ & 0.0005 & -33.1873 \\
\hline $\begin{array}{c}1.95 \mathrm{E}+ \\
03\end{array}$ & 0.0717 & $\begin{array}{c}- \\
22.88696 \\
729\end{array}$ & 0.0014 & -28.6504 \\
\hline $\begin{array}{l}\text { Averag } \\
\text { e value }\end{array}$ & $\begin{array}{c}0.093688 \\
1\end{array}$ & $\begin{array}{c}- \\
21.04788 \\
763 \\
\end{array}$ & $\begin{array}{c}0.0025 \\
27\end{array}$ & -26.8113 \\
\hline $\begin{array}{c}\text { Devian } \\
\text { ce }\end{array}$ & $\begin{array}{c}0.000373 \\
962\end{array}$ & $\begin{array}{c}5.021371 \\
507 \\
\end{array}$ & $\begin{array}{l}8.6687 \\
3 \mathrm{E}-07\end{array}$ & $\begin{array}{c}5.021371 \\
507\end{array}$ \\
\hline
\end{tabular}

Observing the values on the last column of table 5 , we may notice that the values of the electric field intensity and the values of the density of radiated electromagnetic power have a dispersion around the average value of $5.02 \mathrm{~dB}$. 


\section{Conclusions}

After the careful observation of the values presented in the tables, we have noticed that, during measurements with the measuring sensor, in the wave bands $1.95 \mathrm{GHz}$ and $250 \mathrm{MHz}$, different values of the electric field have been registered, more specifically much higher values than the ones of the frequency used by the radio unit $(250 \mathrm{MHz})$. The presentday expansion in the usage of electronic and electric apparatus in every domain has triggered a significant augmentation in the electromagnetic pollution of the environment.

The proper operation of various apparatus and equipment in the new environmental conditions raises complex environmental issues for both designers and users alike. The preoccupation for the research of the electromagnetic fields effects has been completed with the research of its secondary effects, namely the disturbing electromagnetic interferences. Disturbances are produced by the electric field of electric condensers, which trigger unwanted capacitive couplings (disturbances by neighboring field). The transmitting aerials produce disturbances by radiation of the electromagnetic field at large distances in the form of plane waves (disturbances by remote field).

\section{References}

[1] *** - Ecologie electromagnetică-caracterizarea surselor, diagnosticarea efectelorprevenirea şi combaterea lor-Contract 6115 CEEX, 2005 Mataluno

[2] Calotă, S.V., Deliu, N., Licurici, M., Ferastraeru, C., Contulescu, A., Studiu privind expunerea profesională la radiaţii neionizante de înaltă frecvenţă şi riscul pentru sănătate, Institute of Public Health, Bucharest, 1998

[3] Ianoz, M.(Professor at Polytechnics University of Lausanne, Switzerland), Biological and Health Effects of Electromagnetic Fields, conference, Polytechnics University of Bucharest , Faculty of Power Engineering, TICEM Research Centre, march 2003

[4] Zamfirescu, M., Sajin, I., Rusu, I., Sajin, M., Kovacs, E., Efecte biologice ale radiaţiilor electromagnetice de radiofrecvenţă şi microunde, Editura Medicală, Bucureşti, 2000

[5] G., Le radiozioni elettromagnetiche a bordo, in Rivista marittima, anno CXXI Dicembre, Roma, 1988

[6] *** Expunerea omului la câmpuri electromagnetice din domeniul de frecvenţă $10 \mathrm{kHz}-$ $300 \mathrm{GHz}$, Project of Romanian Standard: SR EN 50166-2, IRS, Bucharest (in accordance with the standard EN 50166-2/1994)

[7] *** Romanian Government Resolution GR nr. 59, of 06.09.2006, regarding the minimal security and health requirements when exposing workers to risks generated by electromagnetic fields - Annex: Threshold values for exposure and triggering values for electromagnetic field action;

[8] *** Specific Directive 96/98/EC, referring to the „Maritime Equipment”, compulsory as of the 1 st of January 1999, transposed in Romania by Order of the Minister of Public works, Transports and Housing nr. 582/2003, for the approval of technical norms for equipment and products for maritime vessels, as provided by international cnventions Romania is part of, , code MLPLTL.ANR-EM 2003;

[9] *** Romanian Standard SREN 60945:2001 - Equipments, navigation systems and maritime radiocommunication. General Rules. Trial Methods and Imposed Results;

[10] ***Comparative Analysis of European and American Norms regarding the boundary limits for exposure to an electromagnetic field of the human body in the wave band $\mathrm{O} \mathrm{Hz}$ to $300 \mathrm{GHz}$, the paper „Radio-Freqnency Radiation for Transmitters: A Comparison of U.S. and European Requirements", authors: Steve Dillingham and Nick Cobb; 
[11] ***Romanian Standards SREN 50166-1 şi SREN 50166-2, referrrign to the approved boundary limits of the induced current density and its associated biological effects, the and the boundary limits for the specific absorption rate- SAR, the approved boundary ;imits of the electric frield intensity, the magnetic field intensity and the peak poer density ijn the human body, for controlled and uncontrolled media, withih the wave band $3 \mathrm{kHz}-$ $300 \mathrm{GHz}$. 